\author{
Gabriele Manoli ${ }^{1, *}$, Simone Fatichi ${ }^{1}$, Markus Schläpfer ${ }^{2}$, \\ Kailiang $\mathrm{Yu}^{3}$, Thomas W. Crowther ${ }^{3}$, Naika Meili ${ }^{1,2}$, Paolo Burlando ${ }^{1}$, \\ Gabriel G. Katul ${ }^{4}$, \& Elie Bou-Zeid ${ }^{5}$ \\ ${ }^{1}$ Institute of Environmental Engineering, ETH Zurich, Zurich, Switzerland \\ ${ }^{2}$ Future Cities Laboratory, Singapore-ETH Centre, ETH Zurich, 138602 Singapore \\ ${ }^{3}$ Department of Environmental Systems Science, ETH Zurich, Zurich, Switzerland \\ ${ }^{4}$ Nicholas School of the Environment, Duke University, Durham, NC 27708, USA \\ ${ }^{5}$ Department of Civil and Environmental Engineering, Princeton University, Princeton, NJ 08544, USA \\ *Corresponding author: manoli@ifu.baug.ethz.ch
}

\title{
Magnitude of urban heat islands largely explained by climate and population
}

\begin{abstract}
Urban heat islands (UHIs) exacerbate the risk of heat-related mortality associated with global climate change. The intensity of UHIs is known to vary with population size and mean annual precipitation but a unifying argument is missing, and geographically targeted guidelines for heat mitigation remain elusive. Here we analyze urban-rural surface temperature differences $\left(\Delta T_{s}\right)$ worldwide and find a nonlinear increase of $\Delta T_{s}$ with precipitation that is controlled by water/energy limitations on evapotranspiration and that modulates the scaling of $\Delta T_{s}$ with city size. We introduce a coarse-grained model linking population, background climate, and UHI intensity and we show that urban-rural changes in evapotranspiration and convection efficiency are the main determinants for warming. The direct implication of these nonlinearities is that mitigation strategies aimed at increasing green cover and albedo are more efficient in dry regions, while cooling tropical cities is a challenge that will require innovative solutions.
\end{abstract}

Keywords: Cities, Climate Variability, Green Cover, Population, Urban Heat Islands 


\section{Main}

Cities modify their surface energy balance and generally exhibit higher air and surface temperatures than the surrounding rural areas ${ }^{1-3}$. This phenomenon, known as the urban heat island (UHI) effect, poses a threat to human health as more than half of the world population now lives in cities ${ }^{4}$ and warming can increase morbidity and mortality ${ }^{5,6}$, especially during heat waves ${ }^{7}$. UHIs have been extensively studied in North America ${ }^{2,8}$, Europe $^{9}$, China ${ }^{10,11}$, and globally ${ }^{12,13}$. A link between urbanization-induced warming and city size as measured by its population was first proposed in 1973 based on nighttime air temperature data ${ }^{1}$. With the proliferation of remotely sensed land surface temperature measurements, similar relations have been proposed at the global scale ${ }^{13}$. Local hydroclimatic conditions also contribute to the intensity of UHIs ${ }^{2,14}$, with rising mean annual precipitation causing an increase in urban to rural surface temperature differences $\left(\Delta T_{s}\right)$, a proxy for urban warming with respect to the more efficient cooling of the surrounding rural surfaces. Given the complexity of urban systems, identifying and isolating the causes of UHIs remains challenging ${ }^{3,15}$ and the factors contributing to the observed changes in $\Delta T_{s}$ across city sizes and hydroclimatic conditions continue to be a subject of inquiry and debate $\mathrm{e}^{2,13,14,16}$.

During nighttime, the intensity of UHIs is largely controlled by urban-rural differences in surface geometry, thermal properties, and anthropogenic heat ${ }^{3}$. The causes of daytime $\Delta T_{s}$ are fundamentally different and both changes in convection efficiency associated with surface roughness ${ }^{2}$ and changes in the partitioning of latent/sensible heat fluxes associated with local climate-vegetation characteristics ${ }^{10,14,16}$ have been proposed as the main drivers of warming. Some studies suggested that $\Delta T_{s}$ increases linearly with precipitation due to changes in aerodynamic resistance, as cities in dry climates are more efficient than the barren surrounding in dissipating heat, while the opposite is observed in humid regions ${ }^{2}$. However, the validity of such a linear relation has been questioned. Remote sensing measurements from 32 cities in China hint to the existence of a precipitation threshold above which $\Delta T_{s}$ is insensitive to precipitation changes ${ }^{10}$. In addition, the aerodynamic explanation of UHIs is inconsistent with the observed power law scaling of urban warming with population as an increase in building height (associated with larger city sizes ${ }^{17}$ ) should enhance convection and increase cooling rather than warming. However, the reasoning that "rougher" cities with taller and denser buildings are more efficient in exchanging heat and momentum ${ }^{2}$ is contrary to the observed decrease in roughness length with urban density ${ }^{18}$. Numerical simulations have confirmed possible nonlinear responses of $\Delta T_{s}$ to precipitation ${ }^{16}$ but, unlike previous modeling results, the variability of $\Delta T_{s}$ has been explained by changes in rural temperature ${ }^{16}$ rather than convection efficiency ${ }^{2}$. In short, the causal links 
between $\Delta T_{s}$, population, city texture, and climate appear to be complicated by hidden thresholds and remain uncertain. As a consequence, identifying general guidelines for heat mitigation remains a daunting task ${ }^{15}$ and a fundamental knowledge gap persists in understanding how cooling effects of urban vegetation ${ }^{19}$ and albedo management ${ }^{2}$ vary across cities and climatic conditions. A case in point is the Italian city of Matera which, despite its dense urban fabric and the lowest green cover in Europe (only $0.1 \%$ of the total area ${ }^{20}$ ), exhibits a negative UHI ${ }^{21}$ while Singapore, with more than $50 \%$ of green spaces ${ }^{22}$, shows a daytime $\Delta T_{s}$ of $+1.9^{\circ} \mathrm{C}$ (ref. 21 ). Hence, the efficiency of heat mitigation strategies cannot be direclty inferred from studies on a few selected cities because an adequate basis for generalization is missing. More broadly, such global issues need to be tackled with a holistic perspective to put existing results into geographic context and transfer knowledge across climatic gradients, which frames the scope of this work.

Here, surface temperature anomalies in more than 30000 cities $^{21}$ are analyzed and used to develop a mechanistic coarse-grained model that links $\Delta T_{s}$ to population $(N)$ and mean annual precipitation $(P)$, where $N$ is an aggregate measure for urban infrastructure size and $P$ is a proxy for time-integrated surface-atmosphere exchanges and climatic patterns. The model is based on the fact that, as a city grows, its structure and functioning are predictably modified ${ }^{23}$. Different building materials are employed, heat storage and evapotranspiration fluxes are altered, and human activity and energy consumption increase. The urban fabric (e.g. area, materials, mean building height, height-towidth ratio of street canyons) also changes, thus altering reflectivity and emissivity of the city surface as well as its roughness and convection efficiency relative to the surrounding (often vegetated) areas. Despite the diversity and complexity of urban systems, universal scaling laws linking urban population to infrastructure size and socio-economic metrics exist and have been confirmed when combining data from cities across the entire globe ${ }^{23}$. How can links between such established scaling laws, $\Delta T_{s}$, and climate-vegetation characteristics be beneficially used to globally address urban-induced warming motivates the work here. When coupled to energy and radiative transfer principles, it is shown that the aforementioned scaling laws provide logical bases to coarse-grained representations of UHIs. This approach constitutes a major departure from empirical analysis that lump different mechanisms into statistical correlations, e.g. between $\Delta T_{s}$ and population or urban texture ${ }^{1,12}$. Likewise, it differs from the current state-of-the-science being employed in climate simulations that resolve the physics of energy exchanges and atmospheric flows at the street-canyon and building level but cannot capture emergent large scale phenomena associated with population and infrastructure dynamics. Our findings explain the global variability of UHIs, they complement exisiting micro-scale urban climate studies ${ }^{24}$ and provide guidance for the increasing efforts aimed at greening and cooling world cities, 
especially to the large number of metropolises that have not benefitted from intensive observational or modelling studies. Also, the approach here offers guidance on where detailed observational and simulation studies can be more effective so as to address UHIs across climatic gradients and city sizes. The main novelty of the proposed approach is the inclusion of emergent behaviors of urban-biosphere systems in a coarse-grained model that explains the observed global patterns of $\Delta T_{s}$. These patterns are then translated to general guidelines for planning and retrofitting of cities ${ }^{5,25}$.

\section{Global patterns of urban warming}

The focus of our analysis is on mean daily urban-rural surface temperature differences $\Delta T_{s}$ during summertime when the intensity of UHIs and the risk of heat-related mortality are expected to be the highest ${ }^{8,13}$. Also, any links to precipitation are likely to be more evident during summer beacuse vegetation is active ${ }^{16}$. Consistent with prior results ${ }^{10,16}$ derived from a smaller data set, a nonlinear relation between $\Delta T_{s}$ and mean annual precipitation is found (Fig. 1a). The reported linear increase ${ }^{2}$ holds for low precipitation regimes but $\Delta T_{s}$ saturates at high precipitation values exceeding around $P=1500 \mathrm{~mm} \mathrm{yr}^{-1}$. A nonlinear response between $\Delta T_{s}$ and background temperature $T_{s}$ is also observed (Fig. 1b) with peak warming occurring at $T_{s} \approx 22^{\circ} \mathrm{C}$ and decreasing UHI intensities for warmer climates. A positive correlation between daytime surface UHI intensity and mean air temperature $\left(T_{a}\right.$ between -10 and $30^{\circ} \mathrm{C}$ ) have been reported ${ }^{10}$ suggesting a possible intensification of urban warming under future climate change scenarios ${ }^{26}$. However, an opposite correlation was observed during nighttime ${ }^{10}$ and during the day in $54 \mathrm{US}$ cities $^{27}$. The global results here show that $\Delta T_{s}$ decreases for $T_{s}$ higher than $\approx 25^{\circ} \mathrm{C}$. Unlike previous results suggesting that the scaling $\Delta T_{s} \sim N^{\delta}$ is invariant with climate ${ }^{2}$, precipitation is shown to introduce appreciable corrections to the observed exponent $\delta$ with a weakening of such scaling under wet conditions (Fig. 1c). Specifically, $\delta$ is 0.21 globally but it varies between 0.15 and 0.34 under wet and dry conditions, respectively. These results agree with early work on the impact of soil moisture on the relation between UHI intensity and population ${ }^{28}$ and the values of $\delta$ are in agreement with prior scaling exponents reported in the literature ${ }^{13}$.

The observed global variability of $\Delta T_{s}$ with mean annual precipitation $P$ and urban population $N$ can be expressed mathematically as (see derivation in the Supporting Information, SI):

$$
\Delta T_{s}(P, N)=\frac{1}{f_{s}(P)-\frac{\gamma}{a_{T}} f_{a}(P)} \Delta S(P, N) ;
$$

where $f_{s}^{-1}$ and $f_{a}^{-1}\left[\mathrm{~K} \mathrm{~W}^{-1} \mathrm{~m}^{2}\right]$ represent the surface and air temperature sensitivities to $1 \mathrm{~W} \mathrm{~m}^{-2}$ 
energy forcing, $\gamma$ and $a_{T}$ are phenomenological parameters that account for the coupling between $T_{s}$ and $T_{a}$, and $\Delta S$ [W m ${ }^{-2}$ ] is the energy forcing perturbation due to urban-induced changes in surface albedo $(\Delta \alpha)$, emissivity $\left(\Delta \varepsilon_{s}\right)$, evapotranspiration $(\Delta E T)$, convection efficiency $\left(\Delta r_{a}\right)$, and anthropogenic heat $\left(\Delta Q_{a h}\right)$. Eq. 1 provides a parsimonious description of the coupled urban-biosphere system (Supplementary Fig. S1) based on general scaling laws for urban form/function and global climate relations (see Methods and SI for details). The proposed approach is deemed "coarse-grained" because "fine-grained" properties of cities and rural areas are smoothed over in space and time to focus on collective phenomena and climatic patterns rather than microscopic (i.e., building to block scale) processes. The validity of the model for the purposes of this study can be evaluated by its ability to recover the observed patterns of $\Delta T_{s}$ changes with simultaneous changes in background climate and population (Fig. 1a-c and Supplementary Fig. S2). The model has a good fit and accuracy when predicting the observed trend of global UHIs across precipitation gradients, closely matching the 1:1 line and accounting for $74 \%$ of the variation (inset in Fig. 1a). The agreement between observed and modeled ET (Supplementary Fig. S3) and the modeled impact of background temperature and wind speed on urban warming (Fig. 1b and Supplementary Fig. S4, respectively) are also acceptable, thus confirming the robustness of the approach here. A conceptual analysis of $\Delta T_{s}$ variability using Eq. 1 suggests that the observed nonlinear responses of UHIs to background climate (Fig. 1) arise from distinct mechanisms, the relative contribution of which vary with precipitation ${ }^{2,29}$ as now discussed using the combined data-model results.

The shape of the $P-\Delta T_{s}$ relation is largely controlled by changes in evapotranspiration (ET). In wet climates, energy limitations define an upper bound to ET differences between urban and rural environments while, in arid regions, water limitations reduce the magnitude of rural ET thus limiting the contribution of $\Delta E T$ to $\Delta T_{s}$ (Fig. 1a,d). In dry climates, when the water budget of urban vegetation is supplemented by irrigation, $\Delta T_{s}$ becomes negative creating an "oasis" effect ${ }^{30-32}$. The amount of urban vegetation also plays a role as estimates of urban green cover fractions $\left(g_{c, u}\right)$ from Europe (EU) and South East Asia (SEA) reveal a significant larger green area in cities located in high precipitation regimes (see Methods). This dependence of urban greenery on hydroclimate, together with changes in air specific humidity with precipitation gradients (see results in the SI), explaisn the concavity of the $P-\Delta T_{s}$ relation in Fig. 1a.

As proposed elsewhere ${ }^{2,7}$, urban-rural changes in convection efficiency also contribute to city cooling in dry and warm climates. Given that the height of natural vegetation increases logistically with precipitation ${ }^{33}$, cities in dry regions are aerodynamically rougher than the surrounding rural surfaces characterized by deserts or short vegetation and heat dissipation by convection could be more 
efficient (see Supplementary Fig. S5). Conversely, cities in wet climates are often surrounded by tall forests that exchange heat more efficiently than dense building blocks. In general, the increase in convection efficiency of rural/vegetated surfaces with higher precipitation, increases the energy redistribution factors $f_{s}$ and $f_{a}$ (through the aerodynamic resistance $r_{a}$, see SI) thus damping the impact of urban-rural changes on the magnitude of $\Delta T_{s}$.

Regarding surface albedo, both positive and negative urban-rural differences $\Delta \alpha$ have been reported for single cities ${ }^{3}$, but previous urban research has predominantly focused on cities in temperate mid-latitudes. The new global analysis here suggests that urban albedo has a notable negative dependence on precipitation, and that world cities overall have a higher albedo than the rural surroundings (see Supplementary Fig. S6). Albedo difference therefore contributes to reducing the intensity of UHIs, especially in dry regions where the "oasis" effect is observed. Sparse vegetation associated with low precipitation regimes generates barren rural areas having lower albedo and higher surface temperatures than cities ${ }^{2,34,35}$. This result agrees with a reported daytime cooling of $0.7^{\circ} \mathrm{C}$ associated with a reduction of net radiation loading reported for cities in the Southern United States ${ }^{2}$ and the negative UHIs observed in India during the pre-monsoon summer ${ }^{35}$. Given the observed decrease in background albedo with increasing precipitation, $\Delta \alpha$ contributes to cooling in wet regions but this contribution becomes negligible when compared to the warming effect of $\Delta E T$ and $\Delta r_{a}$ (see Methods). As a global average, precipitation decreases with increasing summer surface temperature above $20^{\circ} \mathrm{C}$ (i.e., not surprisingly precipitation peaks in the tropics where $T_{s}$ is typically in the range of $20-30^{\circ} \mathrm{C}$ throughout the year while $T_{s}$ can exceed $50^{\circ} \mathrm{C}$ in arid regions, see results in the SI) and the modeled $P-\Delta T_{s}$ relation translates into a decrease of UHI intensity with rising background temperature $T_{s}$ (Fig. 1b,e).

Regarding the impact of city size on urban surface warming, the scaling $\Delta T_{s} \sim N^{\delta}$ is largely controlled by changes in convection efficiency and anthropogenic heat fluxes. Compact high-rise buildings dissipate less heat than sparse low-rise structures and anthropogenic release of energy is higher in large dense cities, thus causing the observed increase in urban "skin" temperature with population $N$. However, the scaling exponent cannot be explained by urban fabric and heat release alone as $\delta$ is modified by background climate through changes in evapotranspiration and convection efficiency that depend on precipitation $P$. Our analysis suggests that changes in surface convection efficiency associated with urban density play a key role in regulating the magnitude of surface UHIs (Fig. 1f). This result is in agreement with the fact that, on large spatial and temporal scales, changes in surface roughness and evapotranspiration efficiency are found to have impacts of similar magnitude on surface temperature differences between forested and cleared land ${ }^{29,36}$. 


\section{Heat mitigation strategies}

These findings provide a mechanistic basis for mitigation strategies in different cities around the world, even where the urban climate was not intensively studied. To this purpose, we have analyzed temperature, precipitation, and green cover data for cities in two distinctive climate regions where green cover data were available, i.e. EU and SEA (Fig. 2). Despite large differences in green cover between EU $\left(g_{c, u}=0.07 \pm 0.05\right)$ and SEA $\left(g_{c, u}=0.48 \pm 0.12\right)$, observed $\Delta T_{s}$ values are comparable in the two regions $\left(1.1 \pm 0.6\right.$ and $0.8 \pm 0.9^{\circ} \mathrm{C}$ in EU and SEA, respectively). This evidence questions the effectiveness of increasing efforts aimed at greening global cities to reduce warming under some climatic conditions. Although it could be surprising, such $\Delta T_{s}$ similarity is consistent with the observed nonlinearity in the $P-\Delta T_{s}$ relation that is reasonably predicted by the coarse-grained model. The larger values of precipitation in SEA compared to EU ( $2354 \pm 747$ versus $775 \pm 186 \mathrm{~mm} \mathrm{yr}^{-1}$ ) enhance the contribution of $\Delta E T$ to $\Delta T_{s}$. That is, rural areas in SEA are more efficiently cooled by evapotranspiration due to higher water availability than their EU counterparts, making the goal of minimizing urban-rural temperature differences harder in SEA. Juxtaposition of this finding to climatic zones means that tropical urban environments require a larger extent of green spaces to compensate for the greater reduction in latent heat fluxes caused by urbanization.

A sensitivity analysis of Eq. 1 to changes in urban green cover elucidates this interplay among multiple mechanisms and highlights the fundamental role of background climate for the design of any UHI mitigation strategy (Fig. 3a) by greening. In dry climates, greening can have a substantial cooling effect if urban irrigation is employed ${ }^{19,30}$. In arid regions, rural land surfaces can be warmer than urban areas due to lower albedo, lower convection efficiency, and water-limited evapotranspiration. However, the magnitude of this "oasis" effect is largely controlled by the amount of urban vegetation and the level of irrigation (Fig. 3 and Supplementary Fig. S7). In wet climates, vegetation is not water limited and ET is a dominant component of the rural surface energy balance ${ }^{35}$ so that, to reduce $\Delta T_{s}$, an increasing green cover is needed as $P$ increases (Fig. 3a). Similar nonlinear responses of $\Delta T_{s}$ to changes in urban albedo and population density are found as illustrated in Fig. 3b-c (see also Supplementary Fig. S7, S8). These results suggest that cooling strategies focused on vegetation and albedo are more effective in regions with $P<1000 \mathrm{~mm} \mathrm{yr}^{-1}$ as it is difficult to achieve $\Delta T_{s} \leq 0.5^{\circ} \mathrm{C}$ at higher precipitation regimes. This work also suggests that the impact of population density on $\Delta T_{s}$ is rather small in wet climates when compared to the other factors (e.g., megacities in SEA) but it is maximized in arid regions where $\Delta T_{s}$ can be mitigated by irrigation. Larger efforts or different strategies (e.g., increasing albedo or convection efficiency) are needed in wet climates because the 
replacement of natural vegetation with urban surfaces generates a much stronger contribution to urban warming ${ }^{11,14}$.

\section{Climate-sensitive urban planning}

The importance of urban vegetation as "natural capital" can hardly be disputed ${ }^{37}$ and its significance to provide heat stress relief at the neighborhood scale is well known ${ }^{19}$. However, background climate conditions influence the efficiency of urban vegetation as a city-scale heat mitigation solution. Since urban-rural differences in ET increase with precipitation, under wet conditions almost the entire city area would need to be replaced with green surfaces to substantially decrease $\Delta T_{s}$ (Fig. 3a). Furthermore, vegetation can reduce thermal comfort by increasing air humidity in hot tropical regions ${ }^{38}$, although if it offers shade, it can still significantly enhance pedestrian comfort. Thermal comfort is associated with air and mean radiant temperatures, air humidity, and wind speed rather than surface temperature alone ${ }^{39}$. Hence, while $\Delta T_{s}$ is a good proxy for UHI intensity at the global scale with the advantage of providing a theoretical basis for the factorization of the different mechanisms regulating the surface energy balance ${ }^{7}$, a climate-sensitive design of cities should also account for site-specific urban and climate characteristics as well as air-surface temperature feedback. Our global analysis inevitably sacrifices such fine-scale processes and detailed numerical simulations remain essential to describe the complexity and heterogeneity of real cities from the building to the regional scale ${ }^{40,41}$. High-resolution simulations, however, are computationally expensive, require detailed information about city texture and building material, and municipalities around the world are often called to make planning decisions without any city-specific analysis. Hence, the coarse-grained approach here can provide a first order guideline on expected cooling effects valid across different regions, future climate, and population scenarios to complement micro-scale monitoring and modeling studies. Similarly, the parallel research track of detailed urban energy balance studies ${ }^{24,41,42}$ can improve the presented coarse-grained representation of urban-biosphere interactions by providing refined urban and climate relations.

Given that urban vegetation improves the provision of other ecosystem services (e.g. reduce pollution, improve health, recreation, biodiversity, shading, carbon sequestration, water regulation ${ }^{37,43}$ ) the full extent of its benefits cannot be evaluated based on surface cooling alone. However, it is safe to state that heat mitigation strategies in urban environments experiencing large precipitation should focus on maximizing shading ${ }^{38,44}$ and ventilation ${ }^{45}$ rather than evaporative cooling. As highlighted by previous studies ${ }^{7}$ and confirmed by the results here, the aerodynamic properties of cities also con- 
tribute to regulating the intensity of UHIs. However, how complex, non-uniform, urban geometries influence the exchange of heat and momentum at the land surface is still a subject of open research, especially at the scales that are relevant for urban design ${ }^{40}$. Our analysis confirms that albedo management is also a viable option to reduce warming at the city scale ${ }^{2,46}$ but, given the seasonality of urban warming ${ }^{9}$, albedo modifications can promote winter cooling and increase energy demand, especially in cold regions 41 .

Hence, given the inefficiency of "one size fits all" solutions ${ }^{47}$ and the fact that cities will face higher costs for climate change adaptation due to UHIs ${ }^{48}$, urban planning should be well aware of the nonlinearities discussed here and explicitly incorporate population dynamics and different climatic contexts in the design of heat mitigation strategies. In a recent Environment Strategy, the Mayor of London has set the target of increasing the city's green cover to $50 \%$ by $2050^{49}$. According to our results, this is a reasonable target to reduce warming in a city such as London, that is relatively dry compared to the tropics, but it is not sufficient to cool tropical cities where warming is observed even when the green cover exceeds 50\% (as in the case of Singapore). In warm arid and semi-arid regions, the intensity of UHIs is often negligible or even negative as observed in Matera, which experiences a hot-summer mediterranean climate. Yet, high background temperatures may pose serious risks for public health ${ }^{6}$ and urban vegetation can be beneficial to strengthen negative UHIs further. The need for urban irrigation, however, can cause water scarcity that could be exacerbated in future climate ${ }^{50}$, shifting the anthropogenic pressure on local water resources.

\section{Conclusions}

The science of cities has proceeded through an interplay between novel scaling theories about size and population, energy and radiative conservation principles, aerodynamics, eco-hydrology, and the acquisition of diverse data sources at scales and resolutions unimaginable only three decades ago. Comprehensive analyses aimed at identifying global patterns, trends and complex interactions shaping an urbanizing planet are certainly profiting from such an interplay, as demonstrated by the global analysis here. This study reveals that urban-rural systems exhibit emergent global scale behaviours which can be described by a coarse-grained representation of the underlying social and physical processes. Global climate change and population growth represent some of today's major challenges for cities and our approach offers a novel framework to forecast and mitigate the combined effects of these two stressors on metropolitan areas worldwide. The intensity of UHIs is shown to be nonlinearly modulated by mean annual precipitation and population size with associated changes in heat 
release, albedo, convection efficiency, and evapotranspiration explaining the observed global patterns of urban-rural surface temperature anomalies. City-level strategies aimed at reducing warming should account for these inherent system nonlinearities as local climate-vegetation characteristics influence the efficiency of different cooling solutions being planned now and in the foreseeable future. Cooling the rapidly expanding tropical cities in Africa and South Asia remains a challenge that will require innovative design solutions beyond increasing urban green areas and modifying albedo.

\section{Acknowledgments}

G.M. was supported by the "The Branco Weiss Fellowship - Society in Science" administered by ETH Zurich. E.B.Z. acknowledges support by the US National Science Foundation under grant No. ICER 1664091, the SRN cooperative agreement No. 1444758, and the Army Research Office under contract W911NF-15-1-0003 (program manager Julia Barzyk). The authors would like to thank Peter Edwards, Jan Carmeliet, Christoph Küffer, and Daniel Richards for help and discussions at the beginning of this research. The authors confirm that they have no interest or relationship, financial, or otherwise that might be perceived as influencing objectivity with respect to this work.

\section{Authors contribution}

G.M. designed the study, developed the model and conducted the analysis with contributions from S.F., G.K., and E.B.Z.; K.Y. and T.W.C. analyzed albedo remote sensing observations. G.M. wrote the original draft of the manuscript with inputs from S.F., G.K. and E.B.Z.; M.S., K.Y., T.W.C., N.M., P.B. reviewed and edited the manuscript. All authors discussed the results and contributed to the final version of the manuscript.

\section{References}

1. Oke, T. R. City size and the urban heat island. Atmospheric Environ., 7, 769-779 (1973).

2. Zhao, L., Lee, X., Smith, R. B. \& Oleson, K. Strong contributions of local background climate to urban heat islands. Nature, 511, 216-219 (2014).

3. Oke, T. R., Mills, G., Christen, A. \& Voogt, J A. Urban Climates. (Cambridge Univ. Press, 2017).

4. Grimm, N. B. et al. Global change and the ecology of cities. Science, 319, 756-760 (2008). 
5. Rydin, Y. et al. Shaping cities for health: complexity and the planning of urban environments in the 21st century. Lancet, 379, 2079-2108 (2012).

6. Mora, C. et al. Global risk of deadly heat. Nat. Clim. Change, 7, 501-506 (2017).

7. Zhao, L. et al. Interactions between urban heat islands and heat waves. Environ. Res. Lett., 13, 034003 (2018).

8. Imhoff, M. L., Zhang, P., Wolfe, R. E. \& Bounoua, L. Remote sensing of the urban heat island effect across biomes in the continental USA. Remote Sens. Environ., 114, 504-513 (2010).

9. Zhou, B., Rybski, D. \& Kropp, J. P. On the statistics of urban heat island intensity. Geophys. Res. Lett., 40, 5486-5491 (2013).

10. Zhou, D., Zhang, L., Li, D., Huang, D. \& Zhu, C. Climate-vegetation control on the diurnal and seasonal variations of surface urban heat islands in China. Environ. Res. Lett., 11, 074009 (2016).

11. Liao, W. et al. Stronger Contributions of Urbanization to Heat Wave Trends in Wet Climates. Geophys. Res. Lett., 45, 11310-11317 (2018).

12. Peng, S. et al. Surface urban heat island across 419 global big cities. Environ. Sci. Technol., 46, 696-703 (2011).

13. Clinton, N. \& Gong, P. MODIS detected surface urban heat islands and sinks: Global locations and controls. Remote Sens. Environ., 134, 294-304 (2013).

14. Li, D. et al. Urban heat island: Aerodynamics or imperviousness? Sci. Adv., 5, eaau4299 (2019).

15. Bai, X. et al. Six research priorities for cities and climate change. Nature, 555, 23-25 (2018).

16. Gu, Y. \& Li, D. A modeling study of the sensitivity of urban heat islands to precipitation at climate scales. Urban Clim., 24, 982-993 (2018).

17. Schläpfer, M., Lee, J. \& Bettencourt, L. Urban skylines: building heights and shapes as measures of city size. Preprint at https://arxiv.org/abs/1512.00946 (2015).

18. Grimmond, S. \& Oke, T. R. Aerodynamic properties of urban areas derived from analysis of surface form. J. Appl. Meteorol., 38, 1262-1292 (1999).

19. Gunawardena, K. R., Wells, M. J. \& Kershaw, T. Utilising green and bluespace to mitigate urban heat island intensity. Sci. Total Environ., 584, 1040-1055 (2017). 
20. Eurostat. Urban Europe - Statistics on cities, towns and suburbs doi: 10.2785/91120 (Publications office of the European Union, Luxembourg, 2016).

21. CIESIN. Global Urban Heat Island (UHI) Data Set, 2013. http://dx.doi.org/10. 7927 / H 4 H 7 O CRF (Center for International Earth Science Information Network, 2016).

22. Richards, D. R., Passy, P. \& Oh, R. Impacts of population density and wealth on the quantity and structure of urban green space in tropical southeast asia. Landsc. Urban Plan., 157, 553-560 (2017).

23. Bettencourt, L., Lobo, J., Helbing, D., Kühnert, C. \& West, G. Growth, innovation, scaling, and the pace of life in cities. Proc. Natl Acad. Sci. USA, 104, 7301-7306 (2007).

24. Chrysoulakis, N. et al. Urban energy exchanges monitoring from space. Sci. Rep., 8, 11498 (2018).

25. Sobstyl, J. M., Emig, T., Abdolhosseini Qomi, M. J., Ulm, F. J. \& Pellenq, R. J. Role of city texture in urban heat islands at nighttime. Phys. Rev. Lett., 120, 108701 (2018).

26. Gill, S. E., Handley, J. F., Ennos, A. R. \& Pauleit, S. Adapting cities for climate change: the role of the green infrastructure. Built Environ., 33, 115-133 (2007).

27. Scott, A. A., Waugh, D. W. \& Zaitchik, B. F. Reduced urban heat island intensity under warmer conditions. Environ. Res. Lett., 13, 064003 (2018).

28. Imamura, I. R. Role of soil moisture in the determination of urban heat island intensity in different climate regimes. WIT Trans. Ecol. Envir., 1, 395-402 (1970).

29. Lee, X. et al. Observed increase in local cooling effect of deforestation at higher latitudes. Nature, 479, 384-387 (2011).

30. Oke, T. R. The energetic basis of the urban heat island. Q. J. Royal Meteorol. Soc., 108, 1-24 (1982).

31. Shashua-Bar, L., Pearlmutter, D. \& Erell, E. The cooling efficiency of urban landscape strategies in a hot dry climate. Landsc. Urban Plan., 92, 179-186 (2009).

32. Kumar, R. et al. Dominant control of agriculture and irrigation on urban heat island in India. Sci. Rep., 7, 14054 (2017). 
33. Madani, N. et al. Future global productivity will be affected by plant trait response to climate. Sci. Rep., 8, 2870 (2018).

34. Lim, Y. K., Cai, M., Kalnay, E. \& Zhou, L. Observational evidence of sensitivity of surface climate changes to land types and urbanization. Geophys. Res. Lett., 32, L22712 (2005).

35. Shastri, H., Barik, B., Ghosh, S., Venkataraman, C. \& Sadavarte, P. Flip flop of day-night and summer-winter surface urban heat island intensity in India. Sci. Rep., 7. 40178 (2017).

36. Juang, J. Y., Katul, G., Siqueira, M., Stoy, P. \& Novick, K. Separating the effects of albedo from eco-physiological changes on surface temperature along a successional chronosequence in the southeastern United States. Geophys. Res. Lett., 34, L21408 (2007).

37. Willis, K. J. \& Petrokofsky, G. The natural capital of city trees. Science, 356, 374-376 (2017).

38. Manickathan, L., Defraeye, T., Allegrini, J., Derome, D., \& Carmeliet, J. Parametric study of the influence of environmental factors and tree properties on the transpirative cooling effect of trees. Agric. For. Meteorol., 248, 259-274 (2018).

39. Jendritzky, G., de Dear, R. \& Havenith, G. UTCI - why another thermal index? Int. J. Biometeorol., 56, 421-428 (2012).

40. Llaguno-Munitxa, M. \& Bou-Zeid, E. Shaping buildings to promote street ventilation: A largeeddy simulation study. Urban Clim., 26, 76-94 (2018).

41. Yang, J. \& Bou-Zeid, E. Should cities embrace their heat islands as shields from extreme cold? J. Appl. Meteorol. Climatol., 57, 1309-1320 (2018).

42. Seino, N., Aoyagi, T. \& Tsuguti, H. Numerical simulation of urban impact on precipitation in Tokyo: How does urban temperature rise affect precipitation? Urban Clim., 23, 8-35 (2018).

43. Endreny, T. A. Strategically growing the urban forest will improve our world. Nat. Commun., 9, 1160 (2018).

44. Emmanuel, R., Rosenlund, H. \& Johansson, E. Urban shading - a design option for the tropics? a study in Colombo, Sri Lanka. Int. J. Climatol., 27, 1995-2004 (2007).

45. Wong, M. S., Nichol, J. E., To, P. H. \& Wang, J. A simple method for designation of urban ventilation corridors and its application to urban heat island analysis. Build. Environ., 45, 18801889 (2010). 
46. Akbari, H., Menon, S. \& Rosenfeld, A. Global cooling: increasing world-wide urban albedos to offset $\mathrm{CO}_{2}$. Clim. Change, 94, 275-286 (2009).

47. Georgescu, M., Morefield, P. E., Bierwagen, B. G. \& Weaver, C. P. Urban adaptation can roll back warming of emerging megapolitan regions. Proc. Natl Acad. Sci. USA, 111(8):2909-2914, 2014.

48. Estrada, F., Botzen, W. W. J. \& Tol, R. S. J. A global economic assessment of city policies to reduce climate change impacts. Nat. Clim. Change, 7, 403-406 (2017).

49. Mayor of London. London Environment Strategy https://www. london.gov.uk/ what-we-do/environment/london-environment-strategy (Mayor of London, 2018).

50. Bastin, J. F. et al. Cities of the future, visualizing climate change to inspire actions. Preprint at bioRxiv https://doi.org/10.1101/458018 (2018). 


\section{Figure legends}
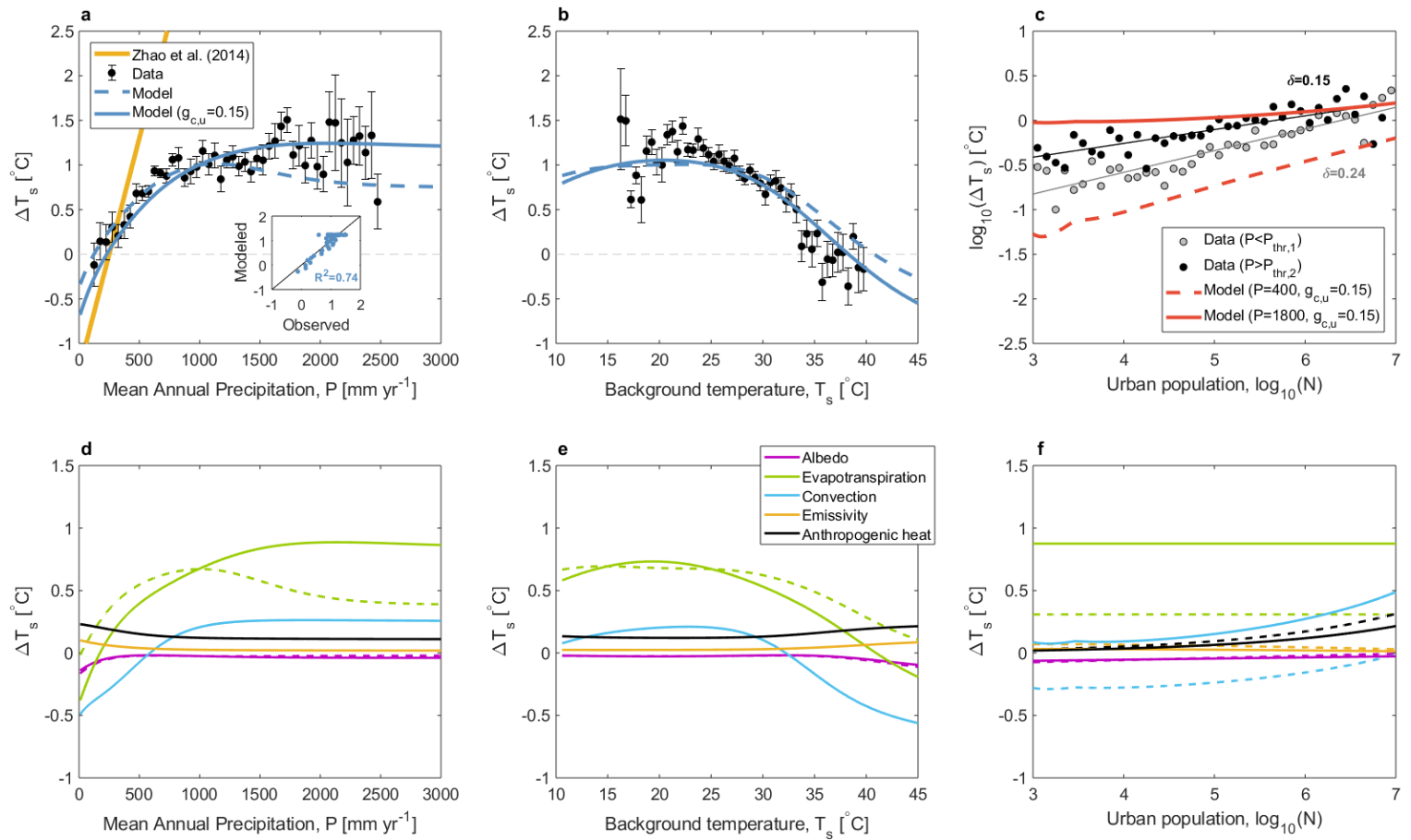

Figure 1: Impact of background climate and population size on urban warming and its components. Observed (markers) and modeled (lines) nonlinear relations between $\Delta T_{s}$ and (a) mean annual precipitation $P$, (b) background temperature $T_{s}$, and (c) urban population $N$. The attribution of $\Delta T_{s}$ to changes in surface albedo $(\Delta \alpha)$, evapotranspiration $(\Delta E T)$, convection efficiency $\left(\Delta r_{a}\right)$, surface emissivity $\left(\Delta \varepsilon_{s}\right)$, and anthropogenic heat $\left(\Delta Q_{a h}\right)$ as a function of (d) $P$, (e) $T_{s}$, and (f) $N$ is also illustrated. A 1:1 comparison of observed and modeled $\Delta T_{s}$ is presented in panel a (inset). The coefficient of determination $\mathrm{R}^{2}$ for this $1: 1$ comparison is also shown. In panels $\mathrm{a}-\mathrm{b}$ and $\mathrm{d}-\mathrm{e}$, model results are featured for a constant urban green cover $g_{c, u}=0.15$ (solid lines) and $g_{c, u}$ proportional to $P$ (dashed lines). Model results are obtained considering an urban irrigation index $I_{r, u}=0.2$ (see SI for details). A linear regression summarizing other data sets for daytime UHIs ${ }^{2}$ is shown for reference (yellow line in panel a). The scaling of $\Delta T_{s}$ with population is shown in panel c-f for wet and dry conditions (solid and dashed lines, respectively). The scaling exponent $\delta$ is calculated by fitting the observations (with $P_{t h r, 1}=700$ and $P_{t h r, 2}=1500 \mathrm{~mm} \mathrm{yr}^{-1}$ ) while the model results are shown for comparison considering two exemplary precipitation levels $\left(P=400\right.$ and $\left.1800 \mathrm{~mm} \mathrm{yr}^{-1}\right)$. Error bars indicate \pm 1 SEM. 

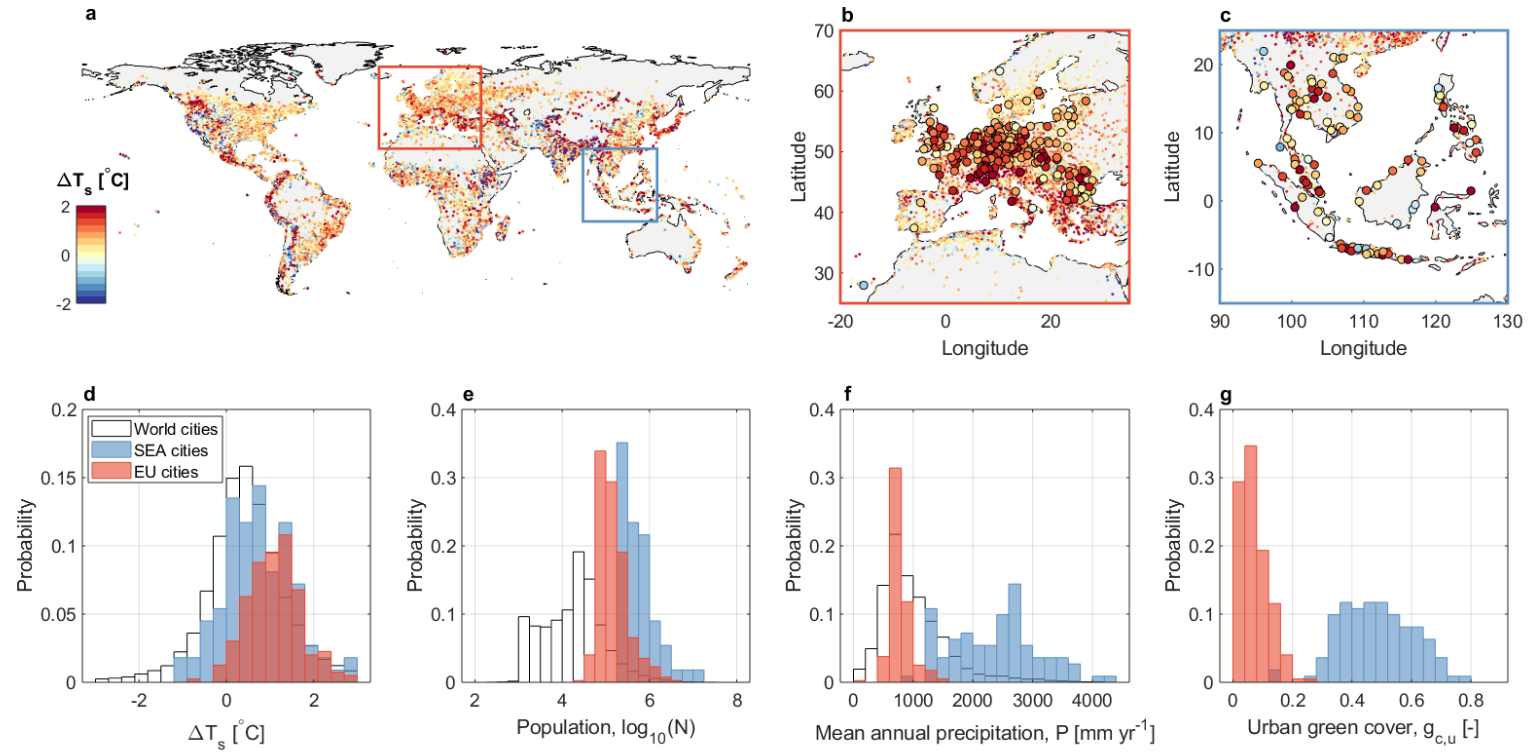

Figure 2: Urban warming and green spaces in Europe (EU) and South East Asia (SEA). Map of summertime UHI intensity in (a) world cities, (b) EU, and (c) SEA. Observed probability distribution of (d) $\Delta T_{s}$, (e) population $N$, (f) mean annual precipitation $P$, and (g) urban green cover $g_{c, u}$. Large circles in panels b-c indicate cities with green cover data ${ }^{20,22}$ used to compute the statistics in panels d-g. 

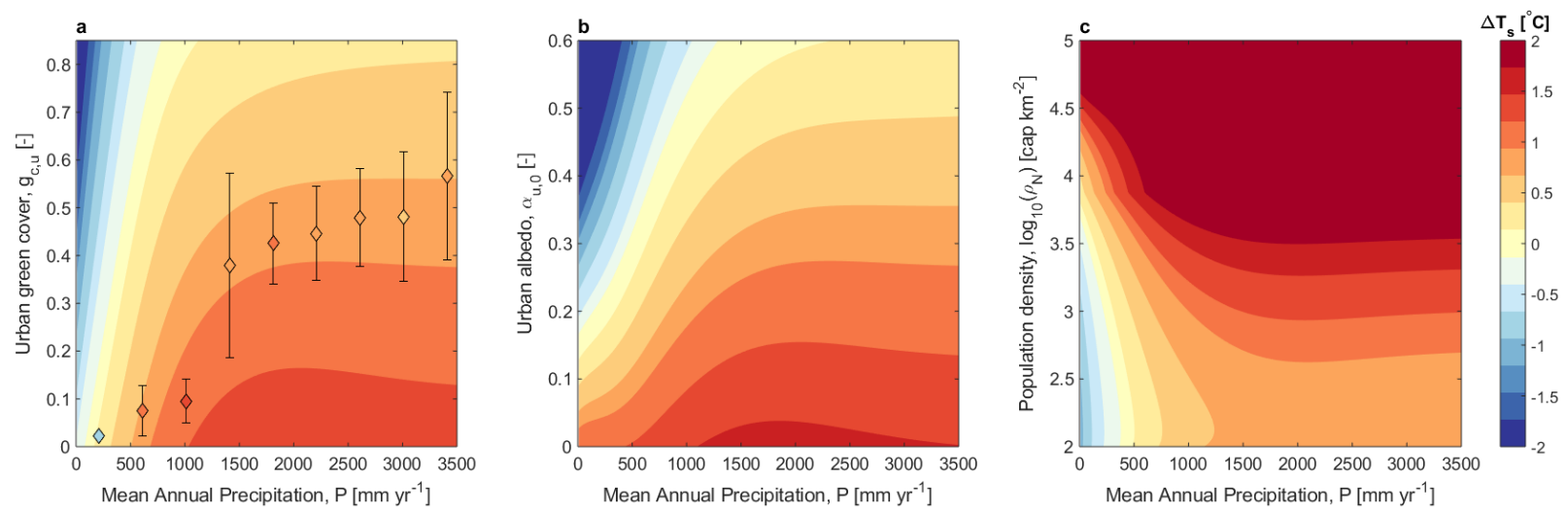

Figure 3: Impact of background climate on the efficiency of heat mitigation strategies. Modeled sensitivity of $\Delta T_{s}$ to changes in (a) urban green cover, (b) urban albedo, and (c) population density. Binned data from EU and SEA cities are also presented in panel a (diamonds). Error bars indicate \pm 1 STD. Results are illustrated for $I_{r, u}=0.2$ and, in the case of panels b-c, for a constant green cover $g_{c, u}=0.15$ (see Supplementary Fig. S7, S8 for the impact of different urban irrigation levels and $g_{c, u}$ values).

\section{Methods}

Urban characteristics. Global estimates of urban-induced changes in surface temperature are obtained from the Global Urban Heat Island Data Set 2013 (ref.21). A surface UHI is defined as the land surface temperature (LST) difference between the urban area and a $10 \mathrm{~km}$ buffer region in the surrounding rural area $\left(\Delta T_{s}\right)$. The dataset includes zonal (i.e., urban and buffer zones) averages of summer daytime maximum and nighttime minimum LST extracted from MODIS LST 8-day composites at $1 \mathrm{~km}$ resolution. This provides daytime and nighttime UHI intensities $\left(\Delta T_{s, d}\right.$ and $\Delta T_{s, n}$, respectively) for more than 30000 cities. Summer is defined as the period between July and August (2013) in the northern hemisphere and January to February (2013) in the southern hemisphere. The urban area is estimated from nighttime lights, settlement points, and their associated population counts in 1990,1995 and $2000^{21}$. Hence, the focus is on urban agglomerates, which may include satellite cities and towns. This ensures the identification of consistent territorial units ${ }^{51}$ and the validity of the urban scaling relations (e.g., between population and urban areas ${ }^{51-53}$ ). In this study, population estimates for the year 2000 are used. Regarding the intesity of UHIs, summertime mean daily conditions are considered so that $\Delta T_{s}=\left(\Delta T_{s, d}+\Delta T_{s, n}\right) / 2$. The choice of daily average temperatures is motivated by two reasons: (i) to be consistent with the focus on climatic patterns and long-term averages (i.e. daytime/nighttime conditions are smoothed over on seasonal timescale); (ii) to ensure 
that all the model assumptions are satisfied (e.g. during daytime/nighttime heat storage effects are typically non-negligible and short-term fluctuations in wind speed and atmospheric stability become more relevant, see model development in the SI). Observed daily and daytime UHI intensities differ in magnitude by $1-2^{\circ} \mathrm{C}$ but they exhibit the same global patterns (see Supplementary Fig. S3) and they fall within the confidence intervals of the model simulations (Supplementary Fig. S2). Thus, for the purpose of this study, daily $\Delta T_{s}$ is considered an appropriate metric of UHI intensity. Similarly, studies on UHIs at climate scales ${ }^{16}$ and heat-related mortality ${ }^{54}$ typically focus on mean daily conditions, although nighttime UHIs can also have significant impacts on public health ${ }^{55}$.

Urban-rural albedo differences are calculated using 16-day shortwave black-sky (BSA) and whitesky (WSA) albedo values extracted from the MODIS albedo product (MCD43B3.005) ${ }^{56}$. The 16-day albedo values at a spatial resolution of $1 \mathrm{~km}$ are used to calculate monthly mean BSA and WSA in urban and buffer areas during the summer of 2013 (using the same urban extent polygons of the global UHI dataset). The monthly mean blue-sky albedo $(\alpha)$ is then determined with the direct radiation ratio and monthly mean BSA/WSA ${ }^{57}$. Note that the albedo of urban surfaces generally varies between 0.09 and $0.27^{3,58}$. Most cities have albedos in the range of $0.20-0.35$ or, in the case of hot regions, $0.30-0.45^{59,60}$ and typical values of urban-rural albedo differences range between -0.09 and +0.03 (with a mean value of -0.05 , ref.3). MODIS observations confirm the overall range but suggest that, globally, the distribution of $\Delta \alpha$ is skewed towards positive values, i.e. cities on average are more reflective than the surrounding (Supplementary Fig. S9). A similar result was found for cities across North America ${ }^{2,14}$. MODIS data also reveal that both urban and rural albedo ( $\alpha$ and $\alpha_{u}$, respectively) decrease with increasing precipitation $P$ (Supplementary Fig. S10). This rural trend can be explained by the increase in forest cover with increasing $P$, while the urban trend can be explained by the more widespread use of white surfaces in hotter and drier climates. A weak decrease of $\alpha_{u}$ with population $N$ is also observed (Supplementary Fig. S10), which is interpreted as the result of shading and radiation trapping mechanisms associated with the 3D structure of cities ${ }^{3,61}$. However, MODIS-derived albedo is biased towards clear sky conditions, observations over cities have numerous uncertainties, and rural values can be influenced by water surfaces and nearby settlements. Therefore, the results here should be considered valid for general global patterns only.

Urban green cover data for 398 cities in the EU and 111 cities in SEA are retrieved from Eurostat ${ }^{20}$ and Richards et al. ${ }^{22}$, respectively (Supplementary Fig. S11). Green urban area and population have a superlinear scaling in EU cities ${ }^{52}$, while a sublinear scaling is found in the tropics (Supplementary Fig. S12). It can be surmised that different "greening" patterns are observed in the two regions due to different climatic and socio-economic factors (e.g. population growth rates, development stage). 
These dissimilarities make it difficult to identify a unique relation linking green space area to urban characteristics at the global scale (Supplementary Fig. S12). Nevertheless, $g_{c, u}$ clearly increases with mean annual precipitation (Supplementary Fig. S13) suggesting that local hydroclimate plays a key role in the amount of urban greenery.

Background climate. Monthly meteorological data of air and surface temperature $\left(T_{a}\right.$ and $T_{s}$, respectively), incoming and net shortwave radiation $\left(R_{s w}\right.$ and $\left.R_{s w, n e t}\right)$, wind speed $\left(W_{s}\right)$, specific humidity of air $\left(q_{a}\right)$, and atmospheric pressure $\left(p_{a t m}\right)$ for year 2013 are retrieved from the Modern Era Retrospective-Analysis for Research and Applications (MERRA) ${ }^{62}$ and used to define background climate conditions (Supplementary Fig.S14). The spatial resolution of MERRA $\left(0.5^{\circ} \times 0.667^{\circ}\right)$ ensures that climatic variables represent the background regional conditions without any influence from urban areas. Rural albedo is computed also from MERRA data as $\alpha=1-\frac{R_{s w, n e t}}{R_{s w}}$ (for comparison with the MODIS albedo product) while specific humidity at saturation $\left(q_{s a t, s}\right)$ is estimated from $T_{s}$ and $p_{a t m}$. Mean annual precipitation $P$ and mean summer precipitation $P_{s}$ are retrieved both from the Global Precipitation Climatology Centre (GPCC) Full Data Reanalysis ${ }^{63}$ and MERRA ${ }^{62}$ (see Supplementary Fig. S15 for a comparison of the two datasets). Data confirm the strong spatial correlation between $T_{s}$ and $T_{a}$ (see Supplementary Fig. S16a which is consistent with temporal correlations illustrated elsewhere ${ }^{9}$ ) and reveal robust relations linking background climate-vegetation characteristics to mean annual precipitation (Supplementary Fig. S16, S17).

Data analysis. To integrate data from different sources (see Supplementary Table S1 for a summary), urban and climate variables are homogenized with the CIESIN dataset considering the coordinates of each city (as latitude/longitude of the centroid of the urban extent). Specifically, meteorological variables retrieved from MERRA are interpolated on the city coordinates using a linear interpolation for 2D gridded data. Green cover data are merged with the CIESIN data considering city names and coordinates (when available). All monthly time series are averaged during summer 2013. The use of multiple data sources introduce uncertainties because of possible discrepancies in methodology and/or urban boundaries. However, this study intentionally focuses on global averages rather than city-specific conditions so that random biases across cities and climates are minimized ${ }^{22}$.

A data binning procedure is employed to identify changes in $\Delta T_{s}$ as a function of $P, T_{s}, W_{s}$ and $N$. To remove the effect of population and analyze only the signal of climate, $\Delta T_{s}$ data are filtered for $N>N_{t h}$, with $N_{t h}=10^{5}$ (thereby reducing the number of cities to 3519). The scaling of $\Delta T_{s}$ with $N$ is determined for dry and wet conditions considering two precipitation thresholds (i.e. $P<P_{t h, 1}$ and $P>P_{t h, 2}$, see Fig. 1c and Supplementary Fig. S18). A sensitivity analysis is performed to assess 
the impact of different precipitation thresholds on the observed scaling (Supplementary Table S2). Given the large number of observations, binned results are illustrated in terms of mean values and related standard error of the mean $\left(S E M=\frac{S T D}{\sqrt{n}}\right.$, where $S T D$ is the standard deviation and $n$ the number of observations).

Results presented in the SI (Supplementary Fig. S19) corroborate previous studies showing that daytime and mean daily $\Delta T_{s}$ values vary with $P$ but nighttime UHIs are not correlated with precipitation $^{2,16}$. Similarly, the observed nonlinear $T_{s}-\Delta T_{s}$ relation does not hold during nighttime (Supplementary Fig. S20). These observations also demonstrate that changes in surface temperature $\Delta T_{s}$ are more sensitive to mean annual precipitation $P$ rather than mean summer precipitation $P_{s}$, confirming that $P$ here has to be interpreted as a proxy of the overall vegetation cover and hydroclimatic conditions of a given region. These are better described by annual precipitation rather than summer precipitation. From a hydrological perspective, this is related to slowly evolving soil water dynamics that regulates ET fluxes during summer seasons ${ }^{64,65}$ and to the existing covariation between annual precipitation and vegetation productivity ${ }^{66}$ that controls the evaporation potential.

Mathematical model. Eq. 1 is derived from the energy balance over a rural land-surface considering urbanization as a perturbation from the rural base state $^{2}$. Model development and parameterization are presented in the SI. Model variables and parameters are listed in Supplementary Table S3, S4. Given the objective of exploring the sensitivity of $\Delta T_{s}$ to as few as possible "summary variables" (i.e. mean annual precipitation $P$ and urban population $N)$ a set of climate relations $\Gamma_{c}=\Gamma_{c}(P)$ linking the meteorological variables $\Gamma_{c}=\left\{T_{a}, T_{s}, \alpha, R_{s w}, q_{s a t, s}, q_{a}\right\}$ to $P$ is derived from fitting background climate data (using the nonlinear least-squares regression in MATLAB, i.e. nlinfit function). A nonlinear relation between the urban vegetation fraction $g_{c, u}$ and $P$ is also derived from EU and SEA green cover data (Supplementary Fig. S13). Urban irrigation is modeled by means of an irrigation index $I_{r, u}$ (Supplementary Fig. S21) that modulates ET varying between 0 (natural conditions) and 1 (no water supply limitations so that ET matches potential evapotranspiration). Changes in urban characteristics with city size are described by scaling laws linking urban area $A_{u}$, mean building height $h_{c, u}$, urban roughness, and urban anthropogenic heat $Q_{a h, u}$ to $N$ (ref. 3,17,23). Previous studies reported a sublinear-to-linear scaling of urban area with population (scaling exponent varying between 0.56 and 1.04, ref. 51,53,67), which is confirmed here at the global scale (estimated exponent of 0.62-0.82, see Supplementary Fig. S22). The mean building height and roughness are employed in the calculation of the aerodynamic resistance $r_{a, u}$ (together with the building density $\rho_{b}$, see Supplementary Fig. S23) and the effective emissivity $\varepsilon_{s, u}$ of the urban surface (through the sky view factor $v_{s k y}$, Supplementary 
Fig. S24). Anthropogenic heat $Q_{a h, u}$ is calculated based on population density ${ }^{3}$, i.e. $\rho_{N}=N / A_{u}$ (Supplementary Fig. S25).

The use of these urban scaling relationships and global climatic trends (Supplementary Table S5, S6) with the surface energy balance provides a novel coarse-grained description of the urban-biosphere system. In analogy with statistical physics, where temperature is a coarse-grained representation of the kinetic energy of a system of microscopic particles, our approach focuses on global space-time averages rather than single cities. Hence, the applicability of the model is limited for specific locations, especially when site characteristics play a dominant role in regulating local microclimate (e.g., topography, ventilation, water bodies). In addition, supplementary results show that, while valid for a wide range of wind speed conditions, the applicability of the simplified approach might be limited at low $W_{s}$ values because the increase in $r_{a}$ causes an increase in UHI intensity associated with lower energy redistribution factors $f_{s}$ and $f_{a}$ (Supplementary Fig. S4). Possible impacts of urbanization on local rainfall generation mechanisms ${ }^{42,68}$ are also neglected. Despite these limitations, when the model assumptions are satisfied (i.e., no local specific conditions) and accurate urban/climate characteristics are available, the model can produce reasonable estimates of city-specific UHI intensities (G.M. et al., in preparation). Note also that the global analysis here focuses on summertime conditions only. This is motivated by (i) the CIESIN data availability that provides a homogenized dataset at the global scale and (ii) the fact that the risk of heat-related mortality is the highest during summer. However, given the observed seasonality of UHIs ${ }^{8,9}$ and its implications for selecting different heat mitigation strategies $^{41,69}$, our coarse-grained approach can be extended to describe the inter-annual variability of $\Delta T_{s}$ (G.M. et al., in preparation). Additional information on the coarse-grained UHI model can be found in the SI.

Model calibration and validation. Model calibration was performed as follows. First we generated a quasi-random set of 11 calibration parameters (see Supplementary Table S3, S4) using the Sobol quasi-random sampling method (function sobolset in MATLAB). Then, we ran Monte Carlo simulations with the generated parameter set (1000 samples) and compared the model results with the observed $P-\Delta T_{s}$ relation (Fig.1a). Calibrated parameters were selected by choosing the simulation with the highest coefficient of determination $\left(R^{2}=0.74\right)$. Model validation was performed by comparing observed and modeled changes in $\Delta T_{s}$ with background temperature $\left(R^{2}=0.81\right)$ and population (Fig.1b-c). Given that the green cover $g_{c, u}$ was considered a calibration parameter but a non-linear relation between urban green cover and precipitation also exists, model performance is assessed considering $g_{c, u}$ both constant and proportional to $P$ (see Supplementary Fig. S2). 
Code availability. The MATLAB code (https : / / www . mathworks. com/products/mat lab. html) of the coarse-grained UHI model is available on Code Ocean (https://doi.org/10. $24433 / \mathrm{CO} .9808462 . \mathrm{v} 1)$.

\section{Data availability}

The Global Urban Heat Island Data Set 2013 is available at https://doi.org/10.7927/ H4H70CRF (accessed on 07/12/2017). MERRA data are retrieved from https: / / disc.gsfc. nasa.gov/daac-bin/FTPSubset2.pl (downloaded on 04/03/2018) while GPCC data are available at https : / / www . esrl . noaa.gov/psd/data/gridded/data.gpcc.html (accessed on 13/09/2016). MODIS albedo data are available at https : / gcmd. nasa . gov/records/ GCMD_MCD 43B3.html (accessed on 15/07/2018). Urban green cover data for EU and SEA cities are available, respectvely, at https: / / ec.europa.eu/eurostat/statistics-explained/ index.php/Urban_Europe___statistics_on_cities,_towns_and_suburbs_-_green_ cities\#Further_Eurostat_information (accessed on 14/06/2017) and https://doi. org/10.1016/j.landurbplan.2016.09.005 (accessed on 29/09/2017). A summary table containing the urban and climate characteristics of the cities analyzed is also made available on Code Ocean.

\section{Methods references}

51. Bettencourt, L. \& Lobo, J. Urban scaling in europe. J. Royal Soc. Interface, 13, 20160005 (2016).

52. Fuller, R. A. \& Gaston, K. J. The scaling of green space coverage in european cities. Biol. Lett., 5, 352-355 (2009).

53. Fang, Y. \& Jawitz, J. W. High-resolution reconstruction of the United States human population distribution, 1790 to 2010. Sci. Data, 5, 180067 (2018).

54. Gasparrini, A. et al. Mortality risk attributable to high and low ambient temperature: a multicountry observational study. Lancet, 386, 369-375 (2015).

55. Clarke, J. F. Some effects of the urban structure on heat mortality. Environ. Res., 5, 93-104 (1972). 
56. Li, Y., Wang, T., Zeng, Z., Peng, S., Lian, X. \& Piao, S. Evaluating biases in simulated land surface albedo from CMIP5 global climate models. J. Geophys. Res. Atmos., 121, 6178-6190 (2016).

57. Chen, D., Loboda, T. V., He, T., Zhang, Y. \& Liang, S. Strong cooling induced by stand-replacing fires through albedo in siberian larch forests. Sci. Rep., 8, 4821 (2018).

58. Oke, T. R. The urban energy balance. Prog. Phys. Geogr., 12, 471-508 (1988).

59. Taha, H., Akbari, H., Rosenfeld, A., \& Huang, J. Residential cooling loads and the urban heat island-the effects of albedo. Build. Environ., 23, 271-283 (1988).

60. Akbari, H., Rosenfeld, A. \& Taha, H. Summer heat islands, urban trees, and white surfaces. In Proc. American Society of Heating, Refrigeration, and Air-Conditioning Engineers, Lawrence Berkeley National Laboratory Report LBNL-28308 (Atlanta, Georgia, 1990)

61. Yang, X. \& Li, Y. The impact of building density and building height heterogeneity on average urban albedo and street surface temperature. Build. Environ., 90, 146-156 (2015).

62. Gelaro, R. et al. The Modern-Era Retrospective Analysis for Research and Applications, Version 2 (MERRA-2). J. Clim., 30, 5419-5454 (2017).

63. Schneider, U. et al. GPCC full data reanalysis version 7.0 at 0.5: Monthly land-surface precipitation from rain-gauges built on GTS-based and historic data. doi: 10.5676/DWD_GPCC/FD_ M_V7_050 (2015).

64. Miguez-Macho, G. \& Fan, Y. The role of groundwater in the Amazon water cycle: 2. Influence on seasonal soil moisture and evapotranspiration. J. Geophys. Res. Atmos., 117, D15114 (2012).

65. Maxwell, R. M. \& Condon, L. E. Connections between groundwater flow and transpiration partitioning. Science, 353, 377-380 (2016).

66. Huxman, T. E. et al. Convergence across biomes to a common rain-use efficiency. Nature, 429 , 651-654 (2004).

67. Bettencourt, L. The origins of scaling in cities. Science, 340, 1438-1441 (2013).

68. Shepherd, J. M. A review of current investigations of urban-induced rainfall and recommendations for the future. Earth Interact., 9, 1-27 (2005). 
69. Taleghani, M., Tenpierik, M., van den Dobbelsteen, A. \& Sailor, D. J. Heat mitigation strategies in winter and summer: Field measurements in temperate climates. Build. Environ., 81, 309-319 (2014). 